\title{
Case Reports of Unusual Presentation of Congenital Diaphragmatic Hernia \\ ${ }^{1}$ Lubna Riaz, ${ }^{1}$ Maida Riaz, ${ }^{1}$ Asfand Tariq, ${ }^{2}$ Muhammad Raahim Ahsan, ${ }^{1}$ Muhammad Aslam \\ ${ }^{1}$ Department of Pediatrics, Shaikh Zayed Hospital, Lahore \\ ${ }^{2}$ Lahore Grammar School, Lahore
}

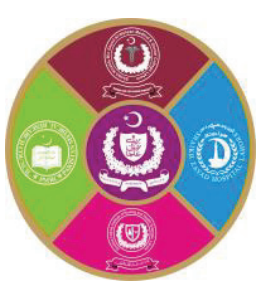

\begin{abstract}
Introduction: Congenital Diaphragmatic hernia usually presents just after birth with respiratory distress and scaphoid abdomen. The most common type occurs through foramen of Bochdalek. Hiatal hernia in neonates, as well as late presentation of congenital diaphragmatic hernia is very rare.

Case Presentation: We report two cases of unusual presentations of diaphragmatic hernias after taking informed consent from the parents. One two-and-a-half-year-old boy presented with repeated chest infection diagnosed as case of Tuberculosis. The second case of neonate with history of reluctance to feed with bulging chest.
\end{abstract}

Conclusion: It is important to identify these presentations, as early correct diagnosis and treatment may be associated with a good outcome, so there is a need for careful clinical examination and proper investigation.

Key words: Diaphragmatic hernia, Late presentation, Foramen of Bochdalek, Good APGAR Score 8/10

\section{INTRODUCTION}

C Congenital Diaphragmatic Hernia occurs in approximately 1 in 2500 births $^{1}$, most of them, occurs posterolaterally through the foramen of Bochdalek. ${ }^{1}$ The usual presentation is at birth with following signs and symptoms:

$\begin{array}{ll}\text { - } & \text { Respiratory distress soon after birth } \\ \text { - } & \text { Tachycardia } \\ \text { - } & \text { Buanosis } \\ \text { - } & \text { Reduing chest wall air entry on either side of chest } \\ \text { - } & \text { Bowel sounds on either side of chest } \\ \text { Scaphoid abdomen }\end{array}$

This abnormalty results when there is an anatomical defect in the diaphragm through which abdominal contents herniate into the chest. There are three types of congenital diaphragmatic hernia, namely Bochdalek, Morgagni and Hiatal. Most common $(70 \%)$ occurs posterolaterally through Foramen of Bochdalek. ${ }^{3}$ Herniation anteriorly (foramen of Morgagni) or through the esophageal hiatus (hiatal) are very rare. The aetiology is not clear but $15 \%$ of the patients may have chromosomal abnormalities (trisomy 13,18 or 21 ) and in $2 \%$ of the cases, it may be familial. ${ }^{4}$ About $10 \%$ of patients present later in life with less severity and a good prognosis. ${ }^{2}$ Early presentation is associated with acute respiratory symptoms and a higher mortality rate, as compared to late presenting diaphragmatic hernias that are associated with chronic gastrointestinal symptoms and a lower mortality rate. ${ }^{6}$

The rate of antenatal diagnosis, nowadays is high, but in developing countries, prenatal detection is rare due to limited facilities. ${ }^{9}$ Congenital diaphragmatic hernia is associated with significant mortality and morbidity ${ }^{8}$ and the severity of this condition depends upon degree of pulmonary hypoplasia and pulmonary hypertension. ${ }^{4}$ So apart from surgery, mechanical ventilation, extracorporeal membrane oxygenation and treatment of pulmonary hypertension may also be required. There have been advances in medical and surgical management of diaphragmatic hernia, but still congenital diaphragmatic hernia has a high mortality rate. ${ }^{10}$

\section{CASE 1}

A two and half years old boy from Lahore presented through pediatric emergency with recurrent history of cough and difficulty in breathing for the last 6 months. He was previously well with no history of fever, trauma, cyanosis and foreign body ingestion. The child was born full term at private hospital in 
Lahore with no hospital admissions during the neonatal period. He was vaccinated according to EPI schedule, there was no significant family history of asthma or history of contact with tuberculosis. During the last six months, the child was treated with multiple oral and intravenous antibiotics, but there was no improvement. On examination, the child was tachypneic with no evidence of cyanosis, clubbing, edema, and normal JVP. He was afebrile with normal heart rate and blood pressure. BCG scar was present, with no lymphadenopathy. He was pale but thriving well. The chest was symmetrical with equal movements bilaterally. Trachea was shifted to right side. Percussion note was resonant bilaterally, but intensity of breath sounds was reduced on left side of the chest with bilateral coarse crepitations. Abdomen was not scaphoid (Fig-1), rest of the examination was normal. X-ray Chest (Fig-2) revealed heterogenous opacities in left upper and middle zones with cyst like radiolucent areas in left side of the chest.

A suspicion of lung cysts or diaphragmatic hernia was made. HRCT chest (Fig-3) confirmed the herniation of bowel loops into the left side of the chest with mediastinal shift to right side, suggestive of left Bochdalek's hernia.

On laparotomy, the herniated contents were reduced into the abdomen with repairing of the diaphragm. The child made a successful recovery and was discharged on 10th post operative day with no complications.

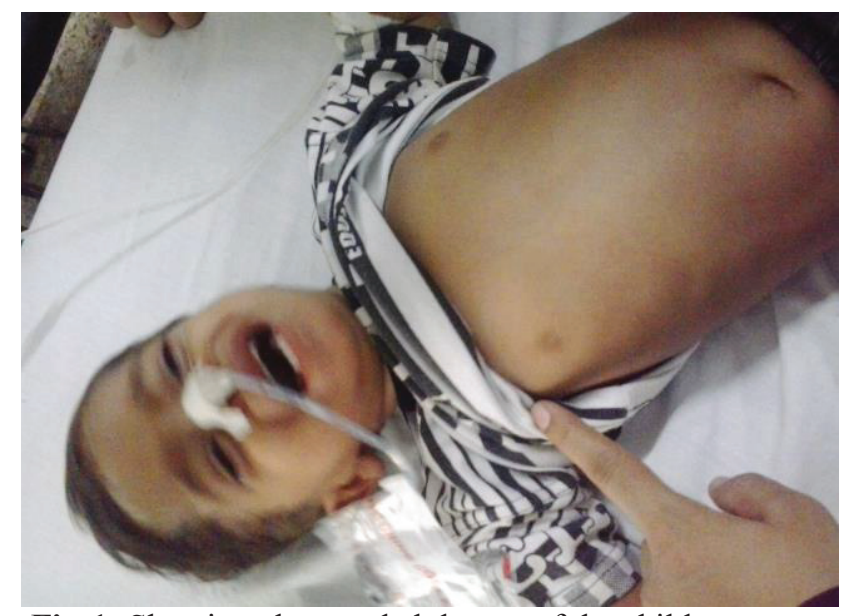

Fig-1: Showing chest and abdomen of the child

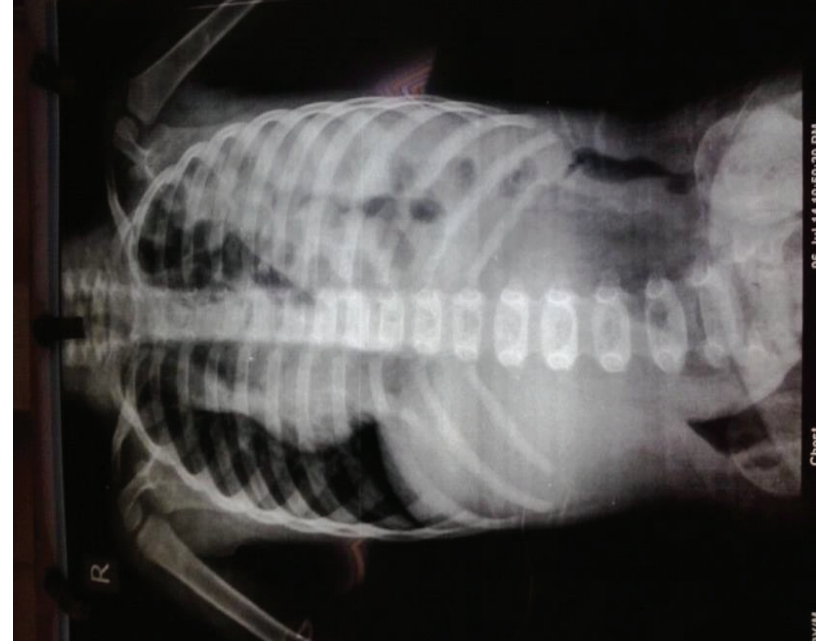

Fig-2: Xray chest and abdomen showing minimal gut shadows in abdomen and cyst like areas in left chest

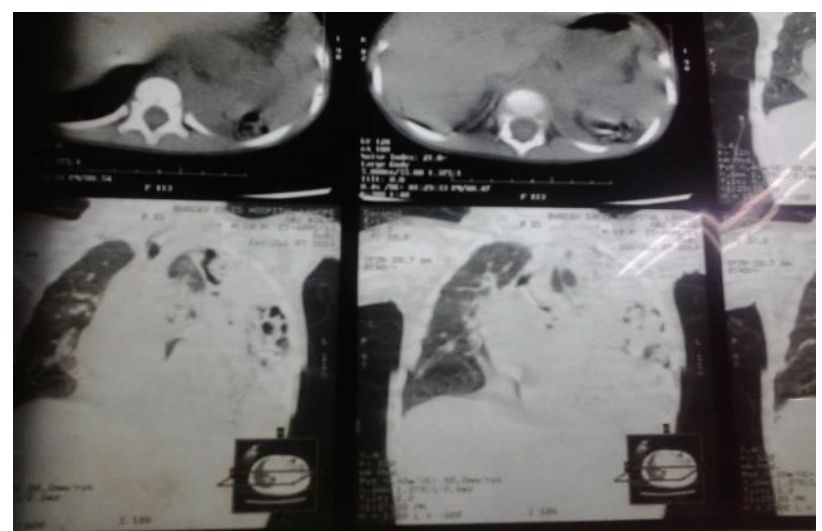

Fig-3: HRCT chest showing gutloop herniation in left chest

\section{CASE 2}

A one day old male baby born through Elective Csection with good APGAR score of 8/10 that is the child was active, pink with no respiratory distress and normal heart rate presented with reluctance to feed. There was no significant antenatal history of maternal infection or PV leaking; the antenatal ultrasound scans were normal. On examination he weighed $3.2 \mathrm{~kg}$, was normal looking, with no cyanosis, respiratory distress or edema. Respiratory rate, heart rate and blood pressure, were normal (Fig-4). Chest seemed to be bulging with reduced breath sounds on right side of the chest. Cardiac examination was normal on auscultation. The shape of the abdomen was not scaphoid. Chest X-ray (Fig5) revealed gut loop shadows in the right side of the chest with mediastinal shift to left side. The lateral view Xray (Fig-6) also showed gut loops traversing the mid portion of diaphragm to reach the thoracic cavity. A diagnosis of diaphragmatic hernia most likely to be Morgagni type, was made. On laparotomy, wide hiatal opening was found, with herniation of gut loops and stomach into the right 
chest. There was no malrotation, and other viscera were normal. The herniated contents were reduced back into the abdomen and diaphragm was repaired. Feeding was initiated at 8th post operative day. The baby was discharged on $12^{\text {th }}$ post operative day with successful recovery.

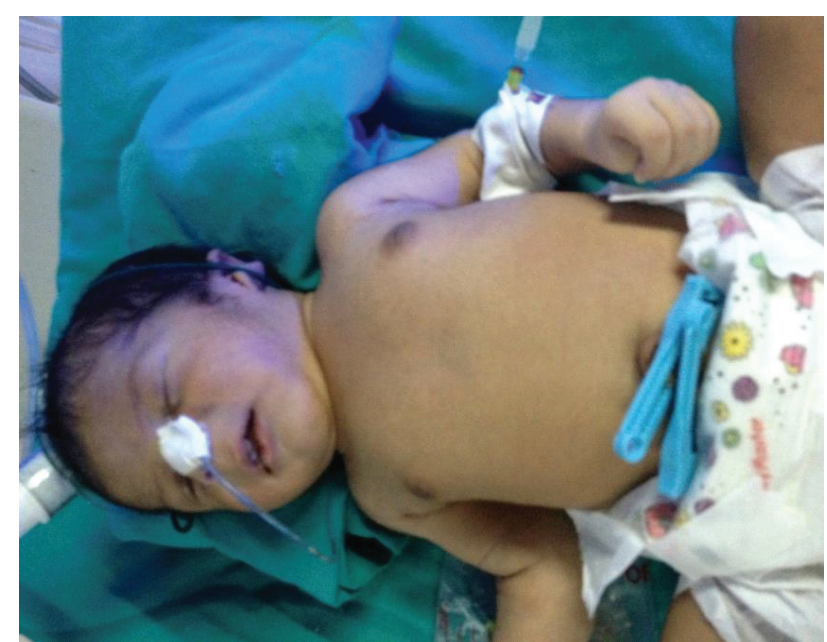

Fig-4: picture of the baby showing mild bulging of chest lacking scaphoid shape.

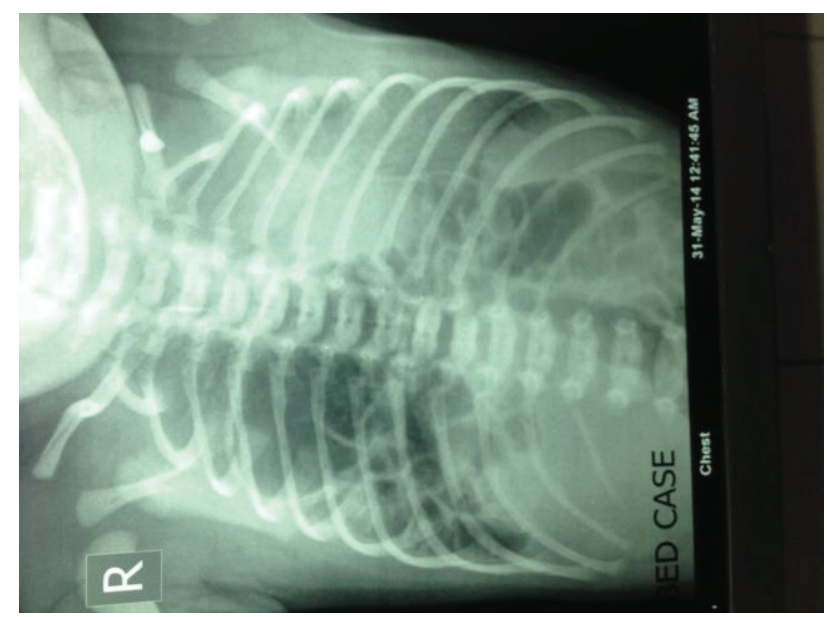

Fig-5: Xray chest showing gut loops in right side of chest

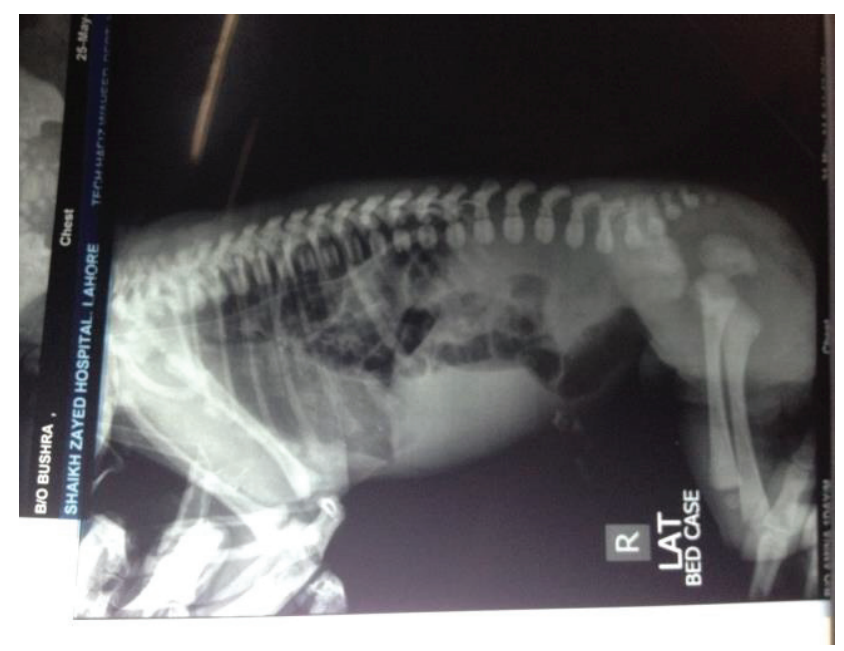

\section{DISCUSSION}

Over $90 \%$ of cases of diaphragmatic hernia are diagnosed antenatally ${ }^{11}$ or within first few hours of life as in our case $2 .{ }^{12}$ However 5 to 30 percent are diagnosed beyond the neonatal period as in case 1 . These cases are misdiagnosed as pneumothorax, consolidation and pleural effusion. ${ }^{13}$ Evidential support from regional studies found the usual presentation of congenital diaphragmatic hernia to be at birth with late presentations reported in a retrospective study, which included the children of more than one month old, admitted in a hospital of Kashmir, India during the period between January 2008 and December 2013. Berman et al in their series of 26 patients reported that 16 patients had early presentation while 4 of them had late presentation. $^{14}$ Dong Jin et al conducted a retrospective study on late presentation of $\mathrm{CDH}$, between January 1981 and June 2012, in a single institute. There were total of 7 patients between the age of 2.5 months to 16 years who presented with nonspecific gastrointestinal or respiratory symptoms and signs. Their diagnosis was made on chest CT and USG. ${ }^{15}$ Our patient (case 1) presented with repeated chest infections, a rare presentation of congenital diaphragmatic hernia at 2.5 years of age, as mentioned in previous study of Srinagar. ${ }^{4}$ There was no family history and the child did not seem to have any chromosomal abnormality. Though late presenting hernias have good prognosis, they still need surgical intervention to relieve the symptoms. ${ }^{6}$ Neonates with congenital diaphragmatic hernia frequently develop hypoxemic respiratory failure. ${ }^{7}$ Our patient (case 2) had no typical respiratory symptoms after birth and was diagnosed on chest X ray. On laprotomy, herniation of stomach and bowel loops through hiatal opening into the chest, was found. Congenital Hiatal hernia is a very rare entity, but treatment still is surgical repair for improving immediate health and prognosis.

\section{CONCLUSION}

This case report emphasizes the need for careful clinical examination and proper investigations keeping in mind the unusual or late presentations of congenital diaphragmatic hernia. Early correct diagnosis and treatment may show a good survival rate. The clinician should have a high index of suspicion for diagnosing such rare congenital anomalies as these can be misdiagnosed and maltreated for Asthma, Tuberculosis or Pneumonia. 


\section{REFERENCES}

1. Van den Hout L, Sluiter I, Gischler S, et al; Can we improve outcome of congenital diaphragmmatic hernia? PediatrSurg Int. 2009; 25(9):733-43.

2. Baglaj $\mathrm{M}$; Late-presenting congenital diaphragmatic hernia in children: a clinical spectrum. PediatrSurg Int. 2004; 20(9):658-69. Epub 2004 Sep.

3. Keijzer R, Puri P. Congenital diaphragmatic hernia. Semin Pediatr Sur 2010; 19: 180-85.

4. Hamid R, Baba A, Shehra A et al. Late presenting congenital diaphragmatic hernia. African J. of Pediatric Surgery. 2014; 11 (2):119-23

5. Hannah K, Peter D B. Congenital diaphragmatic hernia in neonate. Continuing Education in Anesthesia, Critical care and pain. 2005; 5 (5): 171-74.

6. Chang SW, Lee HC, Yeung CY, Chan WT et al.A twenty-year review of early and latepresenting congenital Bochdalek diaphragmatic hernia: are they different clinical spectra? Pediatr Neonatol. 2010; 51(1):26-30.

7. Brendan T. Campbell, Katherine W. Herbst et al. Inhaled Nitric Oxide Use in Neonates with Congenital Diaphragmatic Hernia. Pediatrics. 2014: e420-26.

8. Mary Elizabeth Brindle, Earl Francis Cook et al. A Clinical Prediction Rule for the Severity of Congenital Diaphragmatic Hernias in Newborns. Pediatrics. 2014: e413-19.

9. A. Abubakar, M. Bello, J. Chinda, K. Danladi, and I. Umar, "Challenges in the management of early versus late presenting congenital diaphragmatic hernia in a poor resource setting," African Journal of Paediatric Surgery. 2011; 8(1):29-33

10. C. Doherty and R. J. MacKinnon, "Congenital diaphragmatic hernia - an update," Infant, 2006; 2(6):244-48.

\section{The Authors:}

Dr. Lubna Riaz

Assistant Professor,

Department of Pediatrics,

Shaikh Zayed Hospital, Lahore.

Dr. Maida Riaz

Resident Department of Pediatrics,

Shaikh Zayed Hospital, Lahore.

Dr. Asfand Tariq

Assistant Professor,

Department of Pediatrics,

Shaikh Zayed Hospital, Lahore.

Muhammad Raahim Ahsan

Student,

Cambridge International A Levels,

Lahore Grammar School, Lahore.

Prof. Muhammad Aslam

Department of Pediatrics,

Shaikh Zayed Hospital, Lahore

\section{Corresponding Author:}

Dr. Lubna Riaz

Assistant Professor,

Department of Pediatrics,

Shaikh Zayed Hospital, Lahore.

E-mail: lubnariaz15@gmail.com 